Research Article

\title{
Intelligent Classification Method for Tunnel Lining Cracks Based on PFC-BP Neural Network
}

\author{
Hao Ding $\mathbb{D}^{1},{ }^{1}$ Xinghong Jiang $\mathbb{D},^{2}$ Ke Li $\mathbb{D},{ }^{3}$ Hongyan Guo $\mathbb{D}^{2},{ }^{2}$ and Wenfeng Li $\mathbb{D}^{4}$ \\ ${ }^{1}$ National Engineering Laboratory for Highway Tunnel Construction Technology, Chongqing 400067, China \\ ${ }^{2}$ Chongqing University, Chongqing 400044, China \\ ${ }^{3}$ China Merchants Chongqing Communications Research \&Design Institute Co. Ltd., Chongqing 400067, China \\ ${ }^{4} X i$ 'an Jiaotong University, Xi'an 710049, China \\ Correspondence should be addressed to Xinghong Jiang; jxh_cq@163.com and Ke Li; nick.88@163.com
}

Received 15 September 2020; Revised 11 October 2020; Accepted 6 November 2020; Published 18 November 2020

Academic Editor: Zheng-zheng Wang

Copyright (C 2020 Hao Ding et al. This is an open access article distributed under the Creative Commons Attribution License, which permits unrestricted use, distribution, and reproduction in any medium, provided the original work is properly cited.

\begin{abstract}
Tunnel lining crack is the most common disease and also the manifestation of other diseases, which widely exists in plain concrete lining structure. Proper evaluation and classification of engineering conditions directly relate to operation safety. Particle flow code (PFC) calculation software is applied in this study, and the simulation reliability is verified by using the laboratory axial compression test and 1:10 model experiment to calibrate the calculation parameters. Parameter analysis is carried out focusing on the load parameters, structural parameters, dimension, and direction which affect the crack diseases. Based on that, an evaluation index system represented by tunnel buried depth $(H)$, crack position $(P)$, crack length $(L)$, crack width $(W)$, crack depth $(D)$, and crack direction $(A)$ is put forward. The training data of the back propagation (BP) neural network which takes load-bearing safety and crack stability as the evaluation criteria are obtained. An expert system is introduced into the BP neural network for correction of prediction results, realizing classified dynamic optimization of complex engineering conditions. The results of this study can be used to judge the safety state of cracked lining structure and provide guidance to the prevention and control of crack diseases, which is significant to ensure the safety of tunnel operation.
\end{abstract}

\section{Introduction}

Tunnels are key nodes of road network, so the safety and stability of tunnel structure is the important foundation to guarantee the overall traffic capacity. However, due to the discontinuity, heterogeneity, anisotropy, and other complex characteristics of underground rock mass, it is difficult to meet all requirements in design and construction. Given the plain concrete lining structure lacks reinforcement skeleton and its bending strength is inadequate, it is a commonplace that " $90 \%$ of the tunnels will have leakage and $100 \%$ of the tunnels may have cracks," and diseases such as cracking and water seepage are very prominent [1-3] with the weakening of material performance and the change of external environment during operation. However, as the most common disease and the manifestation of other disease problems, the results of existing evaluation systems targeted to cracks such as specifications, guidelines, and monographs are flawed such as simplex indexes and insufficient consideration of load-bearing characteristics. So, it is imperative to carry out the research on cracked lining under multiple indexes and extract the evaluation indexes and standards based on mechanical principles so as to map out tunnel maintenance plans and transition from an apparent state-determined way to a load-bearing characteristic-determined way $[4,5]$.

In the research of load-bearing capacity characteristics of tunnel cracked lining structure, the causes of tunnel structural diseases are various, presenting complex phenomena and causing different degrees of consequences. Model experiment and monitoring measurement are mainly aimed at specific working conditions, and parameter regularity analysis mainly focuses on finite element calculation. By establishing the physical crack model or setting the contact relationship pattern at the crack position, the load- 
bearing capacity characteristics of cracked lining structure under different load conditions such as plastic ground pressure, loose ground pressure, and unsymmetrical pressure, and the space scale conditions of different crack length, width, depth, and location have been calculated and obtained [5-7]. However, the finite element method has insufficient mechanism in crack tip treatment and crack propagation characteristics, which fails to reflect the crack stability state of the cracked lining structure. The development of algorithms such as extended finite element, boundary element, and discrete element can provide more powerful tools for the detailed study of the mechanical characteristics of cracked lining. Particle flow is a kind of discrete element, which identifies the contact state between particles to calibrate the basic characteristics of a medium. From the perspective of basic particle microstructure, it has prominent advantages of direct-viewing and high efficiency in the analysis and research of crack propagation [8-10].

In the classification evaluation of cracked lining structure, the most commonly used qualitative methods currently are expert scoring method and fault tree analysis, while the quantitative methods are fuzzy comprehensive evaluation method, analytic classification method, and neutral network method [11-13]. Methods such as expert scoring and analytic hierarchy process are simple, easy-to-operate, and are the most commonly used. However, these methods are based on the structural damage performance index, which is greatly influenced by the subjectivity of experts and not directly related to structural load conditions and loadbearing safety, so the evaluation results fail to distinguish structural damage from nonstructural damage of lining and thus the methods mentioned above are unable to provide guidance in disease treatment. The BP neural network has the ability of self-adaptation, self-learning, nonlinear generalization, etc. which is widely used in tunnel construction control, rock-soil deformation prediction, operation disease evaluation, etc. [14-16]. Model experiment and numerical simulation are carried out to obtain initial training data, and the system associating the disease phenomena, load conditions, and stability characteristics through the BP neural network algorithm is established, which proves to be an effective plan combined with the advantages of focused calculation and generalized analysis.

This paper, in response to the problem of lining crack evaluation, uses a PFC particle flow calculation tool to calibrate the calculation parameters through uniaxial compression test and 1:10 large-scale model experiment of cracked lining first. On this basis, parameter analysis is carried out to calculate the bearing stability state and development trend of cracked lining structure under different external effects such as different surrounding rock grades and loading conditions, different design conditions such as lining structure thickness, and different damage states such as crack length, width, and depth, combined with the crack indexes as well as the crack bearing stability and propagation characteristics revealed by calculation results. The BP neural network training data are obtained by the expert scoring method; the tunnel lining crack classification software based on the BP neural network kernel is developed by virtue of
Python programming. The project research combines the advantages of the qualitative macro, detailed, and reliable quantitative calculation of expert evaluation, which provides a new way and idea for tunnel crack classification research.

\section{Bearing Capacity Calculation Method for Cracked Lining}

2.1. Calibration Parameters of Axial Compression Test. The key to numerical simulation of particle flow depends on whether the microparameters of interparticle are reasonably determined. However, given the uncertainty and complexity of interparticle microparameters, there is still no uniform formula to establish the corresponding relationship between the interparticle microparameters and actual macroparameters of materials. The common method at present is to change the micromechanical properties of particle units and their aggregate fabrics, so as to approximate the macromechanical response of materials. In this paper, according to the parameter calibration process proposed by Cundall $[17,18]$, the uniaxial compression numerical simulation experiment is applied and multiple adjustments are made for the microparameters of the model so as to align with the macroproperties of the materials.

The specific process of calibrating the microparameters of C30 concrete material is as follows. In PFC, the contact bond strength model is selected for the numerical uniaxial test. The loaded specimens selected are the standard model specimens adopted by the European Concrete Association (CEB). The diameter of the cylinders is $152 \mathrm{~mm}$ and the height is $305 \mathrm{~mm}$. The calculation model is shown in Figure 1. Through trial calculation and calibration, the calculation parameters are shown in Table 1. Under this condition, the stress-strain curve of concrete is shown in Figure 2. The peak strain of concrete obtained by the simulation test is about 0.0024 , the ultimate strain is about 0.0035 , and the compressive strength is about $23.7 \mathrm{MPa}$, which are consistent with the stress-strain curve of C30 concrete material. In the PFC parameter analysis of mechanical characteristics of cracked lining structure, C30 concrete calculation parameters adopt the values listed in the table.

2.2. Model Experiment Verification. Loading structure method is applied in PFC to calculate the load-bearing capacity characteristics of cracked lining. To ensure the reliability of the calculation results and verify the accuracy of calculation, the load-bearing capacity characteristics of cracked lining are studied in combination with the multifunctional engineering test system for traffic tunnels. According to the system dimensions, the dimensional similarity ratio is taken as $1: 10$, the lining section adopts the section shape of a two-lane highway tunnel, with the lining thickness taken as $40 \mathrm{~mm}$; the rock mass around the lining is filled with grease to lessen the influence of boundary effect and realizes vertical and horizontal uniform loading, with applying load in steps to arrive at the thickness of $27 \mathrm{~m}$ of equivalent soil mass. 


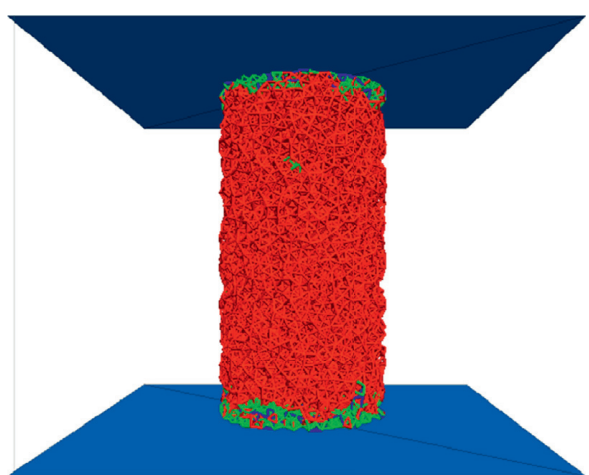

Figure 1: PFC uniaxial compression model.

TABLe 1: Model parameters.

\begin{tabular}{lc}
\hline Parameter & Value \\
\hline Particle radius $(\mathrm{rad} / \mathrm{m})$ & $0.03 \sim 0.05$ \\
Density $\left(\mathrm{des} / \mathrm{kg} / \mathrm{m}^{3}\right)$ & 2040 \\
Equivalent elastic modulus of particles $(\mathrm{GPa})$ & 30 \\
Coefficient of friction, fric & 0.577 \\
Stiffness ratio, $k$ ratio & 2 \\
Parallel bond tensile strength, pb_ten $(\mathrm{Pa} / \mathrm{m})$ & $2.01 e^{7}$ \\
Parallel bond cohesion, pb_coh $(\mathrm{Pa} / \mathrm{m})$ & $15 e^{6}$ \\
\hline
\end{tabular}

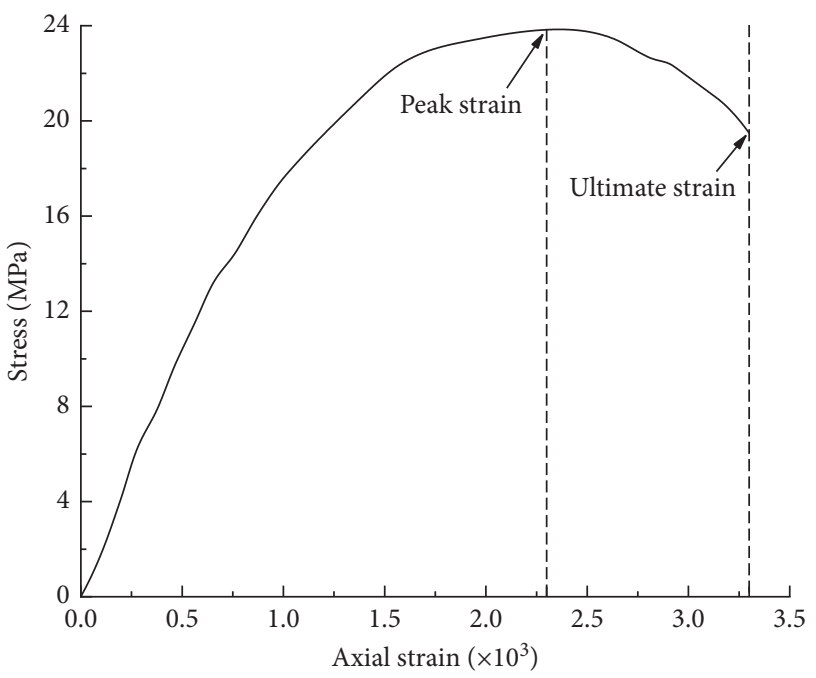

Figure 2: Stress-strain curve of C30 concrete.

The calculation results of the model experiment and the PFC simulation during the loading process are shown in Figure 3. The monitoring results of vault settlement and vault crack depth development are shown in Figures 4 and 5 , respectively. In the loading process, the displacement growth trends of the model experiment and the numerical simulation remain the same, and the numerical values are similar. In addition, the error between the test results and the simulated structure is within $10 \%$, which can be considered that the values taken for the PFC parameters and the simulation calculation can reflect the overall bearing stability of the cracked lining structure in a real scenario. In the loading process, the increasing trends of crack depth at the top arch of the model experiment and the numerical simulation maintain the same; the distribution position and form of structural cracks under simulation conditions are consistent with the results of the model experiment, and the process of equivalent crack depth propagation is similar to the final form. In the loading process, the overall error between the model experiment and the numerical simulation of vault crack depth monitoring position is less than $18 \%$, and the model experiment results are relatively smaller. The main reason is that the crack depth acquisition mainly depends on manual measurement, which is limited by the accuracy and difference of measuring instruments, and there are detailed differences in the specific measurement points under different loads. The measurement results of lining structure cracks are smaller than the actual situation. To sum up, it can be considered that the PFC parameter values are consistent with the simulation calculation and the actual situation, which can reflect the crack propagation law of lining structure under real conditions.

\section{Mechanical Characteristics of Cracked Lining}

3.1. Index Parameter Selection. The load-bearing capacity characteristics of cracked lining structure are related to external loads, structural parameters, and cracking conditions.

Tunnel rock mass environment is complex, and external load modes are various. For ease of calculation and analysis, the surrounding rock grades are used during PFC parameter analysis to comprehensively reflect the load conditions, and the standard method is applied to calculate the load size. Following the experience in tunnel design, the thickness of plain concrete lining structure is normally $30 \mathrm{~cm}, 35 \mathrm{~cm}$, and $40 \mathrm{~cm}$, and in some old tunnels, the thickness may also be $45 \mathrm{~m}$ or $50 \mathrm{~cm}$, so the simulation parameters of lining thickness are taken according to the gradient of $5 \mathrm{~cm}$.

According to the investigation data of nearly 7000 cracks in nearly 50 tunnels on the Shanghai-Chongqing Riverside Expressway, more than $95 \%$ of the lining cracks are less than $10 \mathrm{~m}$ as shown in Figure 6. Therefore, in the PFC calculation, the cracked length parameters taken are $2.0 \mathrm{~m}, 4.0 \mathrm{~m}, 6.0 \mathrm{~m}$, $8.0 \mathrm{~m}$, and $10.0 \mathrm{~m}$. The distribution of lining cracks is extremely uneven, with more cracks in vault, followed by hance, and then side wall in Figure 7. Therefore, five different crack distribution forms, including vault, hance, side wall, vault + hance, and vault + side wall, are taken in the PFC simulation calculation. It is hard to set the initial crack width in the PFC crack simulation calculation. Considering that the crack width is the main manifestation index of crack depth, so it is characterized by different crack depth ratios in the calculation process. In terms of crack direction, given the tunnel structure is dominated by bearing capacity and protection in the circumferential direction, and the influence of longitudinal crack-damage bearing is far greater than that in the circumferential direction, so four angles are set in the simulation calculation in Table 2.

In the table, $H$ represents the width of the tunnel. 


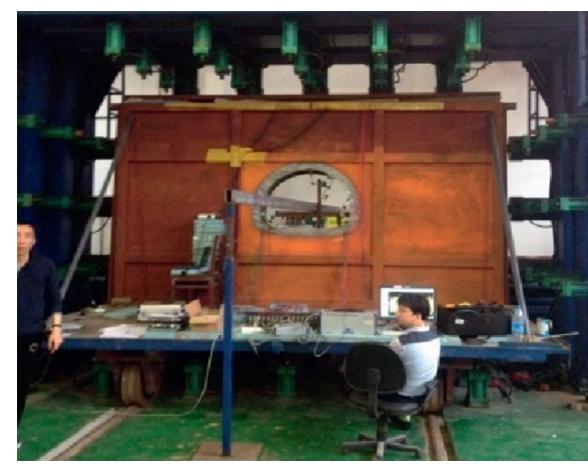

(a)

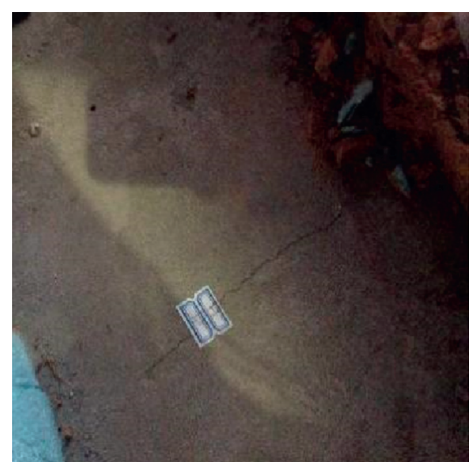

(b)

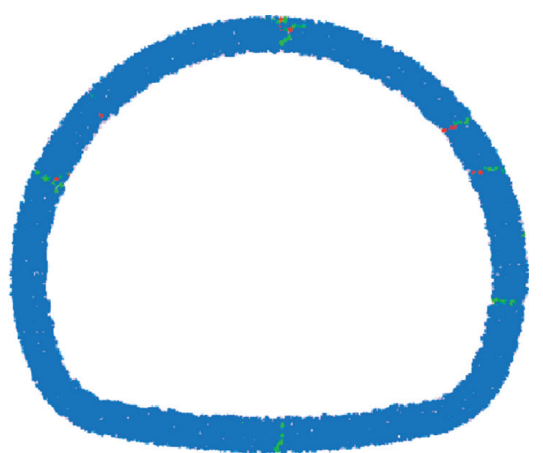

(c)

FIgURE 3: Schematic diagram of model experiment and numerical simulation.

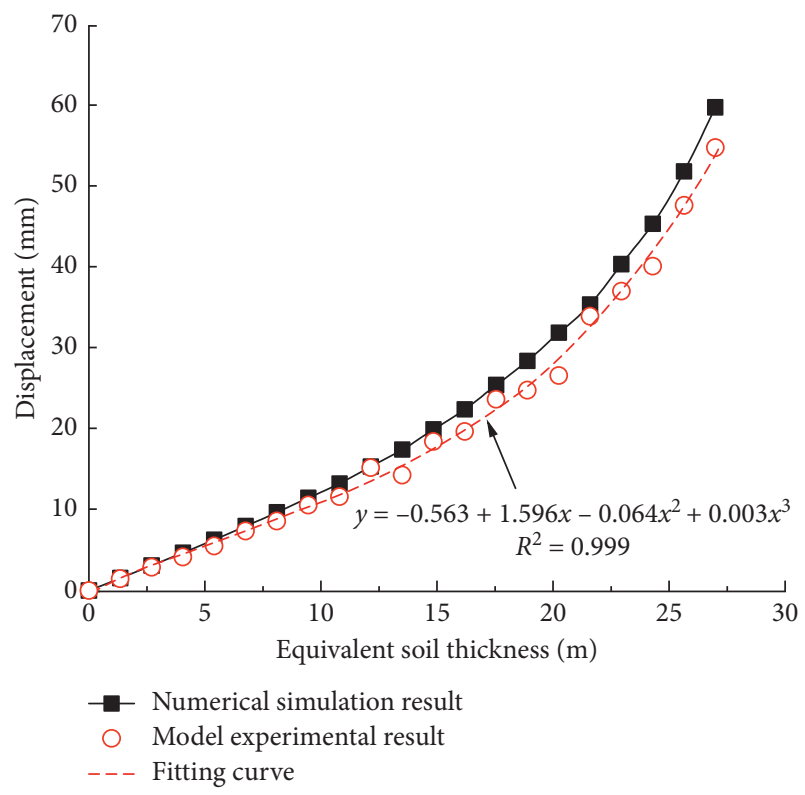

FIGURE 4: Monitoring diagram of lining displacement during loading.

\subsection{Parameter Analysis on Load Condition (Lining Thickness).} The crack depth in the hance is $250 \mathrm{~mm}$, and PFC2D is used to calculate the crack propagation characteristics of the lining structure under deep-buried load condition of different surrounding rock grades (the grade of surrounding rock is determined according to the "Specifications for Design of Highway Tunnels, Section 1, Civil Engineering" (JTG 3370.1-2018), Grade III surrounding rock with a soil column height of $2.7 \mathrm{~m}$, Grade IV surrounding rock with a soil column height of $6.8 \mathrm{~m}$ and Grade V surrounding rock with a soil column height of $13.5 \mathrm{~m}$, Grade III surrounding rock lining with a thickness of $30 \mathrm{~cm}$, Grade IV surrounding rock lining with a thickness of $40 \mathrm{~cm}$ and, Grade V surrounding rock lining with a thickness of $50 \mathrm{~cm}$ ). The simulation results are shown in Figure 8. Under the action of different surrounding rock loads, when the structure cracked, the derivative cracks are similar in position and degree. Slight cracks appear in the vault, side wall, and the

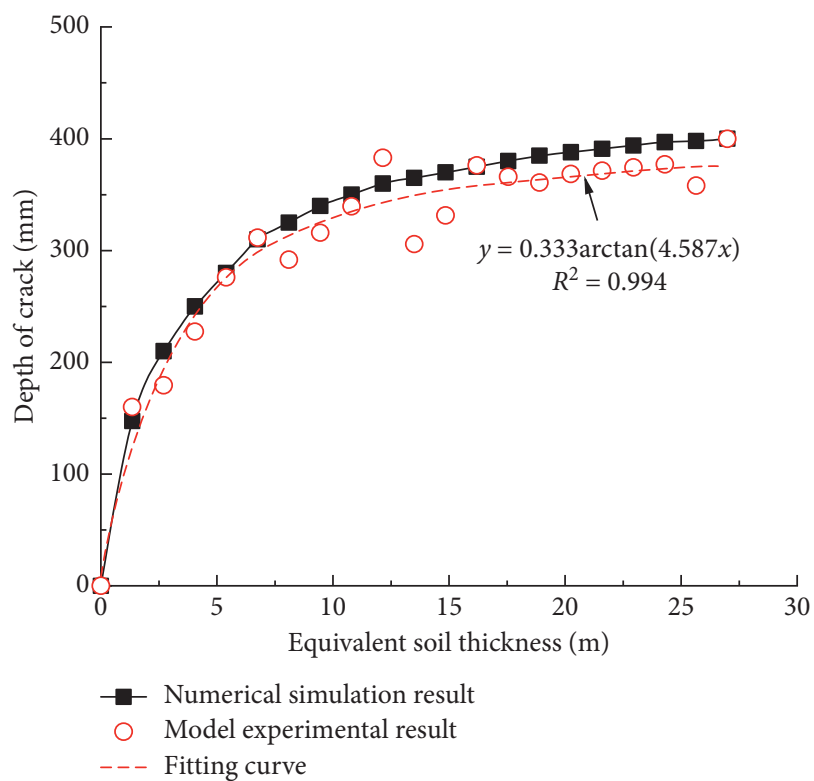

FIgURE 5: Curve of lining vault depth propagation during loading.

middle of invert, and the inner and outer sides of cracks have not been connected, or regional large-scale cracks have not been formed, causing the overall instability or collapse of the structure. It can be preliminarily judged that when the surrounding rock condition is poor, the strengthening of the lining structure design can partially offset the structural adverse effects caused by the increased load, and the surrounding rock grade exerts less influence on the mechanical characteristics of the cracked lining in this type of supporting structure.

Green represents intact lining structures, blue for tensile cracks, red for shear cracks, and yellow for preset cracks. The calculation results remain the same for all subsequent PFC2D.

3.3. Parameter Analysis on Cracked Position. For Grade V surrounding rock with the lining thickness of $50 \mathrm{~cm}$ and the crack depth of $3 / 4 \mathrm{~h}$, when there exist different cracks in the 


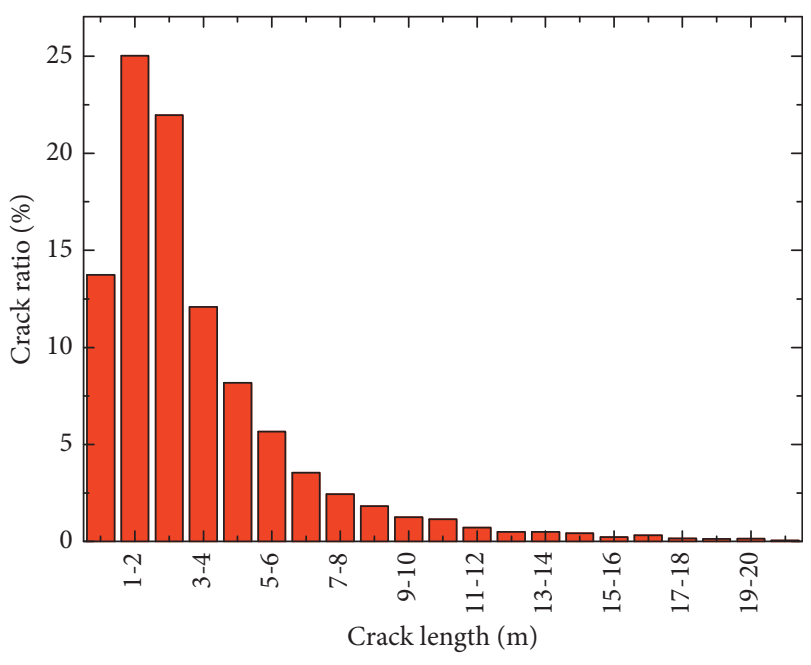

$\square$ Crack ratio

FIGURE 6: Investigation results of crack length.

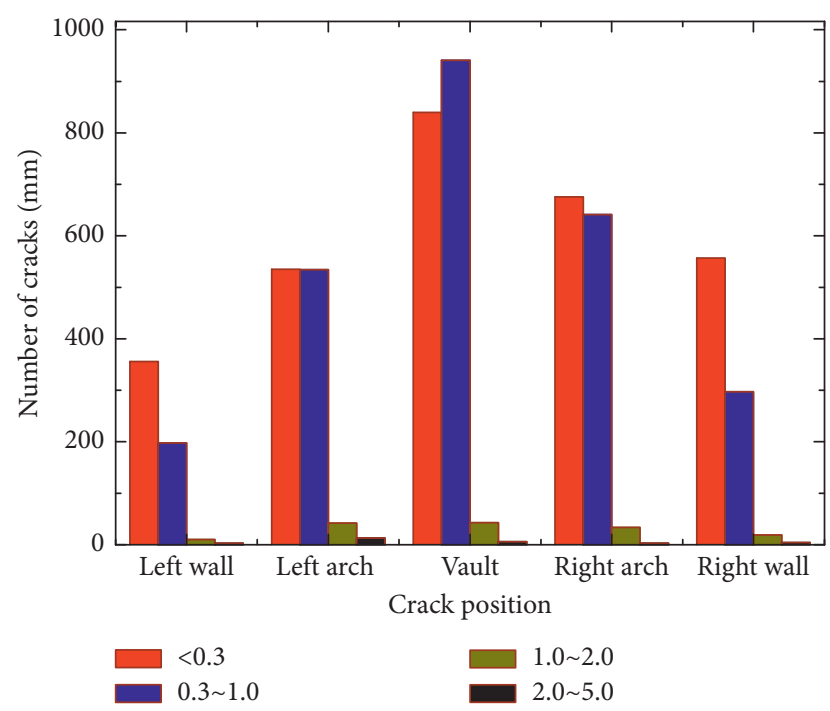

FIGURE 7: Investigation results of crack position.

vault, hance, and side wall, the final PFC2D calculation results of structural damage distribution are shown in Figure 9. Under the action of vault cracks, the derivative cracks are mainly tensile cracks, and there are secondary cracks on both sides of the vault, the hance, the bottom of the side walls, and the invert, forming a cluster of cracks in the arch with a small number of shear cracks on the outside, and the arch structure is likely to slide and fall. Under the action of hance cracks, derivative cracks appear in the vault, the hance, and the middle of the invert. The cracks are mainly shear cracks, which are distributed both inside and outside the vault and hance, and the structural risk is mainly the collapse and falling of the lateral blocks where the cracks are close to the arch. Under the action of side wall cracks, when secondary tensile cracks appear in the arch and the opposite side wall, shear cracks appear in the opposite side wall. The structural risk lies in the damage to the arch wall on the opposite side by cracks.
3.4. Parameter Analysis on Crack Depth. For Grade V surrounding rock with the lining thickness of $50 \mathrm{~cm}$, the PFC2D calculation results of lining structure crack derivative state under the action of different crack depths are shown in Figure 10. Under the action of cracks at $1 / 4 H$ of the vault, there are secondary cracks in the vault, hance, and invert, and the main types of cracks belong to tension crack. When the crack depth increases to $2 / 4 \mathrm{H}$, cracks occur at the bottom of the side wall, and the type of the new cracks belongs to tension crack. When the crack depth increases to $3 / 4 H$, the number of cracks in the vault increases, and new cracks occur in the left and right vaults. The main types of the new cracks are attributed to tension crack, with shear cracks existing in some areas outside the structure. The difference in tunnel crack depth may lead to the increase of the number and distribution of secondary cracks in the structure, especially in the arch, where the risk of peeling, chip off-falling, and even collapse is greatly increased.

3.5. Parameter Analysis on Crack Length. For Grade V surrounding rock with the lining thickness of $50 \mathrm{~cm}$, when the crack depth is $3 / 4 \mathrm{~h}, \mathrm{PFC} 3 \mathrm{D}$ is used to calculate the influence of different crack lengths on the load-bearing characteristics of the lining structure. In the calculation process, the longitudinal length of the structure is $10 \mathrm{~m}$, which is the length of the lining structure of Model-1 tunnel. The simulation results are shown in Figure 11. When the crack length is $5 \mathrm{~m}$, a small number of tensile cracks appear in the arch of lining structure under the action of load. A large number of arch tensile cracks appear when the crack length increases to $6 \mathrm{~m}$. When the crack length increases to $7 \mathrm{~m}$, shear cracks begin to occur in the hance on the opposite side. When the crack length increases to $8 \mathrm{~m}$, the cracks in the hance on the opposite side penetrated. It can be concluded that, if the tunnel Model-1 is taken as a load-bearing unit and the crack depth is large, the structural stress is closely related to the length.

Red represents the intact lining structure, green for tensile cracks, blue for the shear cracks, and yellow for preset cracks. The calculation results are the same for all subsequent PFC3D.

3.6. Parameter Analysis on Crack Direction. For Grade V surrounding rock with the lining thickness of $50 \mathrm{~cm}$, when the crack depth is $3 / 4 \mathrm{~h}$, the PFC $3 \mathrm{D}$ calculation results of lining structure crack derivative state under the action of different crack directions (longitudinal and $45^{\circ}$ oblique) are shown in Figure 12. It can be seen that longitudinal cracks have a greater impact on the load-bearing safety of the structure by comparison and analysis of the distribution and number of cracks. Under the action of longitudinal cracks, structural damage occurs in the arch and arch walls on both sides of the structure, and there exist both tensile and shear cracks of derivative cracks, so the structural integrity is relatively poor. Under the action of oblique cracks, the structural derivative cracks are mainly distributed in the envelope area of vault longitudinal direction and crack 
TAвLE 2: Table for selection of influencing factors and calculation level of bearing capacity of cracked lining structure.

\begin{tabular}{lcccccccc}
\hline Level & $\begin{array}{c}\text { Surrounding rock } \\
\text { grade, } R\end{array}$ & $\begin{array}{c}\text { Tunnel buried } \\
\text { depth, } H_{t}\end{array}$ & $\begin{array}{c}\text { Lining thickness, } \\
T(\mathrm{~cm})\end{array}$ & $\begin{array}{c}\text { Cracked } \\
\text { position, } P\end{array}$ & $\begin{array}{c}\text { Length, } L \\
(\mathrm{~m})\end{array}$ & $\begin{array}{c}\text { Width, } W \\
(\mathrm{~mm})\end{array}$ & $\begin{array}{c}\text { Depth, } \\
D\end{array}$ & $\begin{array}{c}\text { Direction, } A \\
\left({ }^{\circ}\right)\end{array}$ \\
\hline 1 & III & $0.5 H$ & 30 & Vault & 2.0 & 1.0 & 0 & 0 \\
2 & IV & $1.0 H$ & 35 & Hance & 4.0 & 2.0 & $1 / 4 \mathrm{~h}$ & 22.5 \\
3 & V & $1.5 H$ & 40 & Side wall & 6.0 & 3.0 & $2 / 4 \mathrm{~h}$ & 45 \\
4 & VI & $2.0 H$ & 45 & Vault +hance & 8.0 & 4.0 & $3 / 4 \mathrm{~h}$ & 67.5 \\
5 & - & $2.5 H$ & 50 & Vault + side wall & 10.0 & 5.0 & $4 / 4 \mathrm{~h}$ & 90 \\
\hline
\end{tabular}

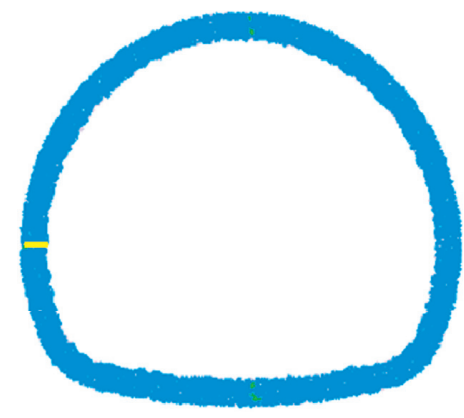

(a)

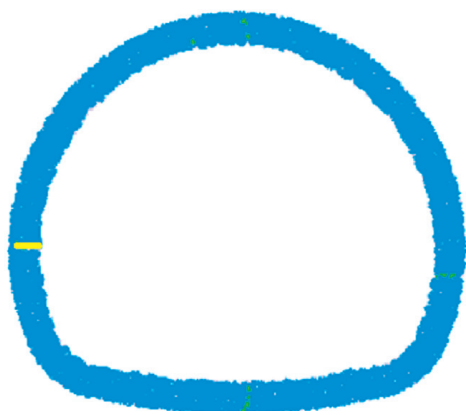

(b)

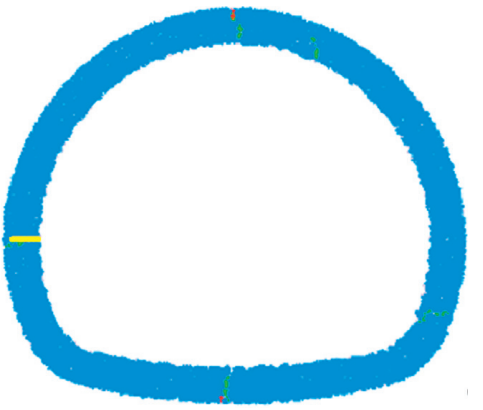

(c)

FIGURE 8: Crack-damage propagation characteristics of lining structure under different loads (thicknesses). (a) Grade III surrounding rock (30 cm thick). (b) Grade IV surrounding rock $(40 \mathrm{~cm}$ thick). (c) Grade V surrounding rock (50 $\mathrm{cm}$ thick).

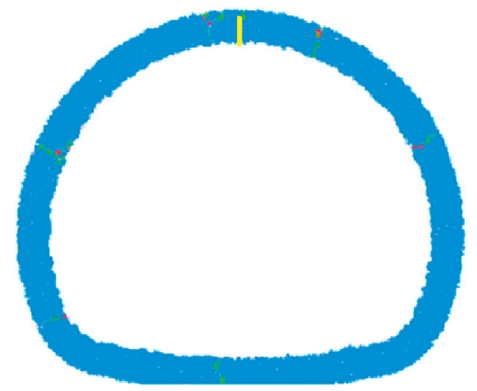

(a)

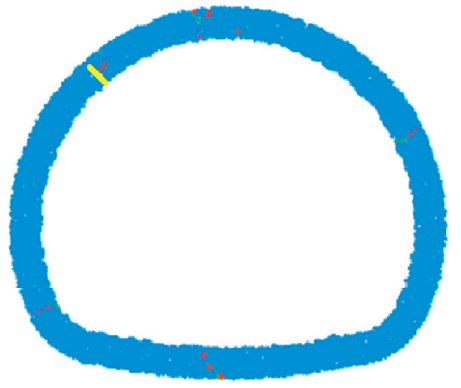

(b)

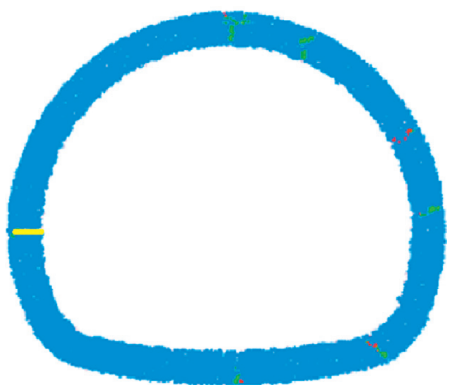

(c)

FIGURE 9: Crack-damage propagation characteristics of lining structure at different crack positions. (a) Vault crack action. (b) Hance crack action. (d) Side wall crack action.

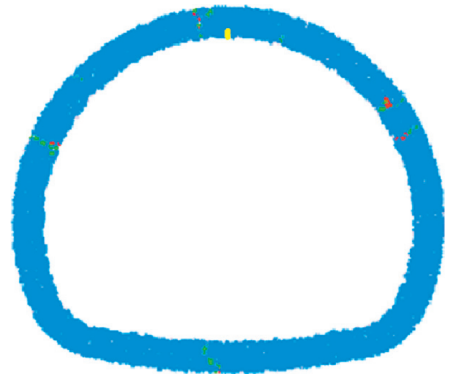

(a)

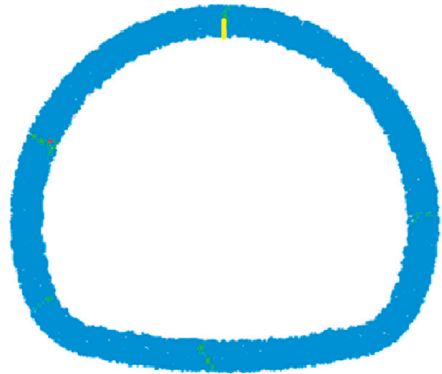

(b)

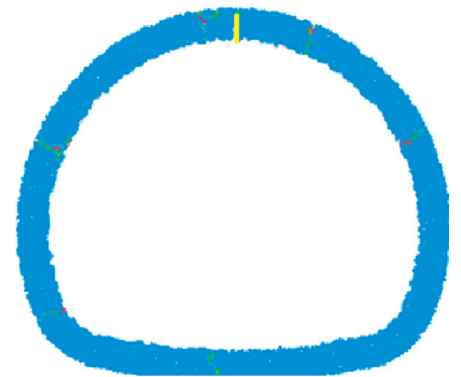

(c)

FiguRE 10: Crack-damage propagation characteristics of lining structure under different crack depths. (a) Crack depth: 1/4H. (b) Crack depth: $2 / 4 H$. (c) Crack depth: $3 / 4 H$. 


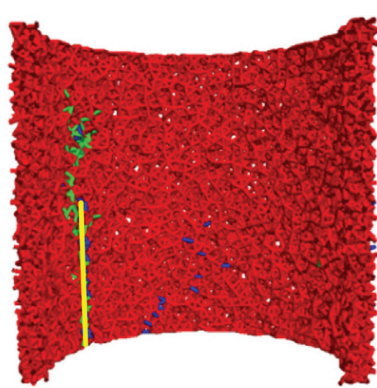

(a)

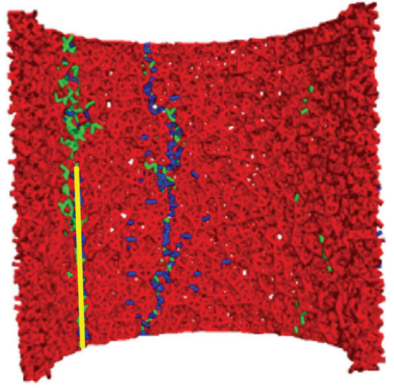

(b)

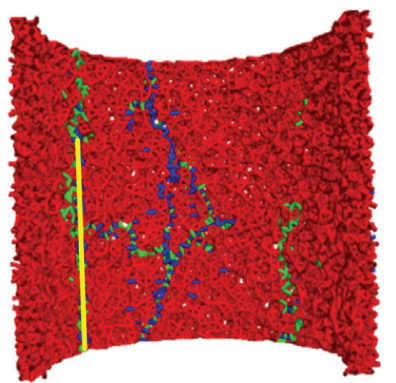

(c)

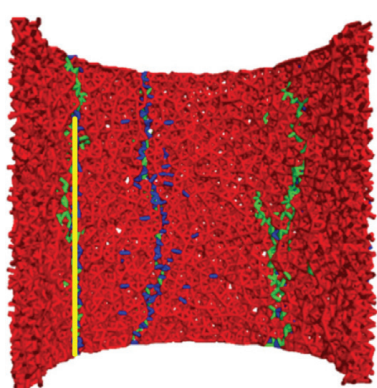

(d)

FIGURE 11: Crack-damage propagation characteristics of lining structure under different crack lengths. (a) $5 \mathrm{~m}$ long crack. (b) $6 \mathrm{~m}$ long crack. (c) $7 \mathrm{~m}$ long crack. (d) $8 \mathrm{~m}$ long crack.

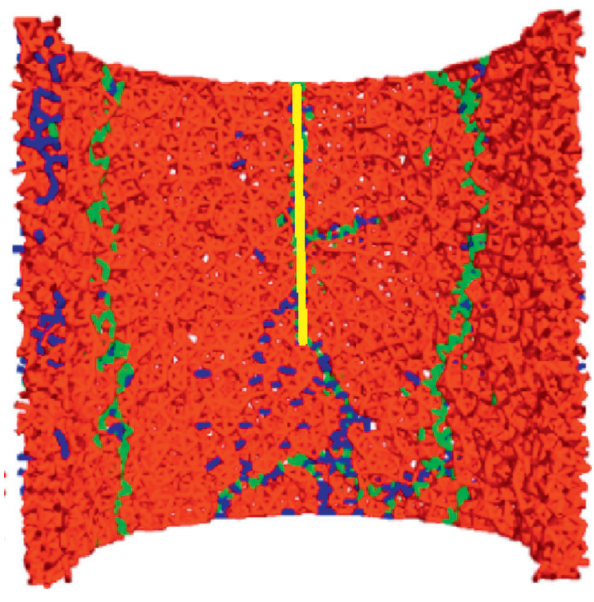

(a)

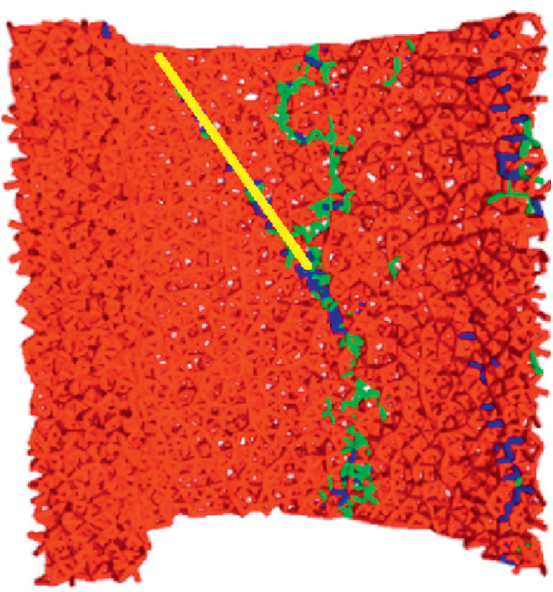

(b)

FIGURE 12: Crack-damage propagation characteristics of lining structure in different crack directions.

direction, and the influence of structural integrity is relatively small.

\section{Classification of Tunnel Cracks}

4.1. BP Neural Network. BP neural network is one of the most commonly used neural networks, whose full name is the artificial neural network based on the error back propagation algorithm [18]. The BP neural network is composed of input layer, hidden layer, and output layer. When the number of neurons in hidden layer is enough, the BP neural network with a three-layer structure can well approximate a discontinuous function with limited parameters. When there are $i$ inputs, $j$ hidden layer neurons, and $k$ outputs, the output of the hidden layer is

$$
P_{j}=f\left(\sum_{i} w_{i j} x_{i}-b_{j}\right),
$$

where $w_{i j}$ is the connection weight between the input layer and the hidden layer, the input variable, the threshold of the hidden layer, and also the activation function of the hidden layer. The S-shaped function is often used as the activation function for the hidden layer, which is mainly divided into log-sigmoid function and tan-sigmoid function. Although both of them encounter the gradient disappearance, compared with log-sigmoid function, tan-sigmoid output is centered on 0 and converges fast. At the same time, tansigmoid function has been successfully applied in the nonlinear prediction [19]. Therefore, this paper chooses tansigmoid function as the activation function of the hidden layer, and its expression is

$$
f_{\tan -\operatorname{sig}}=\frac{2}{1+e^{-2 t}}-1
$$

where $t$ is the input variable of the hidden layer. The output of the output layer is

$$
S_{k}=g\left(\sum_{j} w_{j k} P_{j}-b_{k}\right),
$$

where $w_{j k}$ is the connection weight of the hidden layer and output layer, $b_{k}$ is the threshold of the output layer, and $g$ is the transfer function of the output layer. In this paper, the linear function, purelin function, is selected as the transfer function of the output layer, and its expression is 


$$
g_{p}=a,
$$

where $a$ is the input variable of the output layer.

4.2. Parameters of BP Neural Network Variables. The loadbearing characteristics of cracked plain concrete lining structure mainly include two surrounding rock parameters (surrounding rock grade $R$ and tunnel buried depth $H_{t}$ ), and one lining structural dimension parameter (lining thickness), and five crack information parameters (crack position $P$, crack length $L$, crack width $W$, crack depth $D$, and crack direction $A$ ). Due to the strong correlation between the design thickness of lining structure and the surrounding rock grade, the worse the surrounding rock condition is, the thicker the structure is. According to the calculation and analysis, the enhancement of corresponding structure thickness design under different surrounding rock grade conditions makes it able to resist the load increased by the change of surrounding rock, and the ultimate crack-damage bearing characteristics are similar. Therefore, in BP neural network training, the surrounding rock grade and lining structure thickness are not considered as the amount of training.

To sum up, the comprehensive evaluation of crack lining performance is characterized as follows:

$$
P_{x}=f\left(H_{t}, P, L, W, D, A\right) .
$$

4.3. Crack Classification Sample. The training data of the BP neural network are mainly calculated from crack bearing characteristics. Through modeling and calculation, the loadbearing characteristics data of cracked lining under 471 sets of working conditions are obtained. For each set of calculation results, they are divided into four grades according to the degrees of influence, which are denoted as 1, 2, 3, and 4, respectively. The crack meanings are the same as I, II, III, and IV in Table 3. Combined with PFC numerical calculation results and the expert scoring system, the initial data of the 471 lining crack conditions and the comprehensive rating are determined. When the training data of the BP neural network are sorted out, different tunnel depths are characterized by $0.5,1.0,1.5,2.0$, and 2.5 , respectively, vault, hance, side wall, vault +hance, and vault + side wall are characterized by $1,2,3,4$ and 5 , respectively, and the relative depths of cracks are characterized by $0,0.25,0.50 .75$, and 1.0 , respectively. Due to the limits of textual length, some parts of the training data are extracted after, as shown in Table 4.

\subsection{Data Set Division and Effect Evaluation}

4.4.1. Data Division. In this paper, a BP neural network model is established by using 471 sets of data samples. Through the stratified sampling method, the data are divided into 10 parts according to the attributes of data source. Then, Kennard-Stone algorithm is used to divide each part of data into two parts according to the ratio of $4: 1.80 \%$ of the samples in each part are combined as the training set and $20 \%$ as the test set.

When using the model for training and testing, the parameters are normalized to the interval of $[0,1]$, and the final output value of the model is transformed into the actual order of magnitude value through antinormalization. In the setting of the network parameter, the number of iterations is 2000 , the target accuracy is $1 \times 10^{-5}$, and the maximum number of failures in the verification set is 200 (this parameter does not exist in the Bayesian regularization algorithm).

4.4.2. Evaluation Indexes. Mean square error $\left(E^{2}\right)$ and determination coefficient $\left(R^{2}\right)$ are used as evaluation indexes:

$$
\begin{aligned}
& E^{2}=\frac{1}{n} \sum_{i=1}^{n}\left(p_{i}-\widehat{p}_{i}\right)^{2}, \\
& R^{2}=1-\frac{\sum_{i=1}^{n}\left(p_{i}-\widehat{p}_{i}\right)^{2}}{\sum_{i=1}^{n}\left(p_{i}-\bar{p}_{i}\right)^{2}}
\end{aligned}
$$

where $n$ is the number of data samples; $p_{i}$ is the measured value; $\widehat{P}_{i}$ is the predicted value; and $\bar{P}$ is the average value of measured data.

4.4.3. Number of Neurons. To compare the influence of different neuron numbers and training algorithms on the prediction performance of the BP neural network, L-M algorithm, quasi-Newton algorithm, and Bayesian regularization algorithm are used to compare the prediction performance, and each training algorithm is used to predict the hidden layer neurons at three levels $(6,7$, and 8$)$, respectively. Among them, the middle level selection with 7 neurons in the hidden layer is based on the empirical formula $2 m+1$, where $m$ is the number of neurons in the input layer. In order to avoid the influence of network initial weight and threshold randomness, each combination of BP neural networks continuously runs for 10 times, and the 10time mean value of evaluation indexes is selected for evaluation. The predicted $E^{2}$ mean values of the training set and test set are shown in Figure 2.

It can be seen that, under the same training algorithm, different numbers of hidden-layer neurons have an impact on the prediction accuracy of the training set and the test set. According to the comparison, the $E^{2}$ of each training algorithm is greater when the number of hidden layer neurons is 6 and 8 than when the number of hidden layer neurons is 7. Therefore, 7 is the optimal number of neurons in the hidden layer, L-M algorithm, quasi-Newton algorithm, and Bayesian regularization algorithm [20-22] shown in Figure 13 , and it is used to carry out fitting process, having achieved more optimal prediction performance. The $E^{2}$ predicted for the training set is $2.021 \times 10^{-10}, 2.378 \times 10^{-10}$, and $2.132 \times 10^{-10}$, respectively, and the $E^{2}$ predicted for the test set is $1.512 \times 10^{-10}, 1.994 \times 10^{-10}$, and $1.257 \times 10^{-10}$, respectively. 
TABLE 3: Judgment of fitting result grade of the BP neural network.

\begin{tabular}{|c|c|c|}
\hline $\begin{array}{l}\text { Crack } \\
\text { grade }\end{array}$ & $\begin{array}{l}\text { Fitting } \\
\text { result }\end{array}$ & Engineering significance \\
\hline $\bar{I}$ & $0.8 \sim 1.2$ & The cracks are stable without growth \\
\hline II & $1.8 \sim 2.2$ & $\begin{array}{c}\text { The degree of crack damage is deepening, but the cracks have not yet penetrated along the vertical and horizontal } \\
\text { directions. New cracks form in different positions }\end{array}$ \\
\hline III & $2.8 \sim 3.2$ & $\begin{array}{c}\text { New cracks form in different positions. Some cracks have penetrated, but there is no concentrative penetration } \\
\text { of multiple cracks. }\end{array}$ \\
\hline IV & $3.8 \sim 4.2$ & Multiple cracks penetrate concentratively. \\
\hline
\end{tabular}

TABLE 4: BP neural network training sample data.

\begin{tabular}{|c|c|c|c|c|c|c|c|}
\hline Simulation number & Buried depth, $H_{t}$ & Cracked position, $P$ & Length, $L(\mathrm{~m})$ & Width, $W(\mathrm{~mm})$ & Depth, $D$ & Direction, $A\left({ }^{\circ}\right)$ & Crack grade \\
\hline 1 & 0.5 & 1 & 6.0 & 3.0 & 0.5 & 0 & 2 \\
\hline 2 & 2.5 & 1 & 6.0 & 3.0 & 0.5 & 0 & 4 \\
\hline 3 & 1.5 & 3 & 6.0 & 3.0 & 0.5 & 0 & 1 \\
\hline 4 & 1.5 & 5 & 6.0 & 3.0 & 0.5 & 0 & 4 \\
\hline 5 & 1.5 & 1 & 2.0 & 3.0 & 0.5 & 0 & 1 \\
\hline 6 & 1.5 & 1 & 10.0 & 3.0 & 0.5 & 0 & 3 \\
\hline 7 & 1.5 & 1 & 6.0 & 1.0 & 0.5 & 0 & 2 \\
\hline 8 & 1.5 & 1 & 6.0 & 5.0 & 0.5 & 0 & 4 \\
\hline 9 & 1.5 & 1 & 6.0 & 3.0 & 0 & 0 & 1 \\
\hline 10 & 1.5 & 1 & 6.0 & 3.0 & 1 & 0 & 4 \\
\hline 11 & 1.5 & 1 & 6.0 & 3.0 & 0.5 & 45 & 2 \\
\hline 12 & 1.5 & 1 & 6.0 & 3.0 & 0.5 & 90 & 1 \\
\hline 471 & 1.5 & 1 & $\begin{array}{l}\cdots \\
6.0\end{array}$ & 3.0 & 0.5 & 0 & 3 \\
\hline
\end{tabular}

4.4.4. Training Algorithm. When the number of neurons in the hidden layer is 7 , it can be seen from Table 5 that the $R^{2}$ predicted by the three training algorithms for both the training set and the test set is greater than 0.95 , showing good accuracy of prediction. The $R^{2}$ for the training set and the test set by the L-M algorithm is 0.958 and 0.982 , respectively. The prediction accuracy is higher than that by the quasi-Newton algorithm and is slightly lower than that by Bayesian regularization algorithm. Among them, the Bayesian regularization algorithm shows the best prediction performance, and the $R^{2}$ for the training set and the test set was 0.976 and 0.989 , respectively. The Bayesian regularization algorithm which has adaptive regularization parameters can better avoid the underfitting and overfitting issues in network training, and then guarantee the robustness and generalization performance of the BP neural network. In this paper, the Bayesian regularization algorithm-based BP neural network with the hidden layer neuron being 7 is established.

4.5. Crack Classification System. Through data training, the BP neural network has been able to fit the classification characteristics of cracked lining perfectly. During engineering application, in order to facilitate practical operation within the range of reliability requirements, the calculation result grade of the BP neural network is judged according to Table 3, and the bearing capacity and stability of cracked structure gradually weaken from Grade I to Grade IV. If the fitting result is within the table range, the expert system can be introduced for comprehensive judgment. The judgment data as the training data are updating continuously, thus

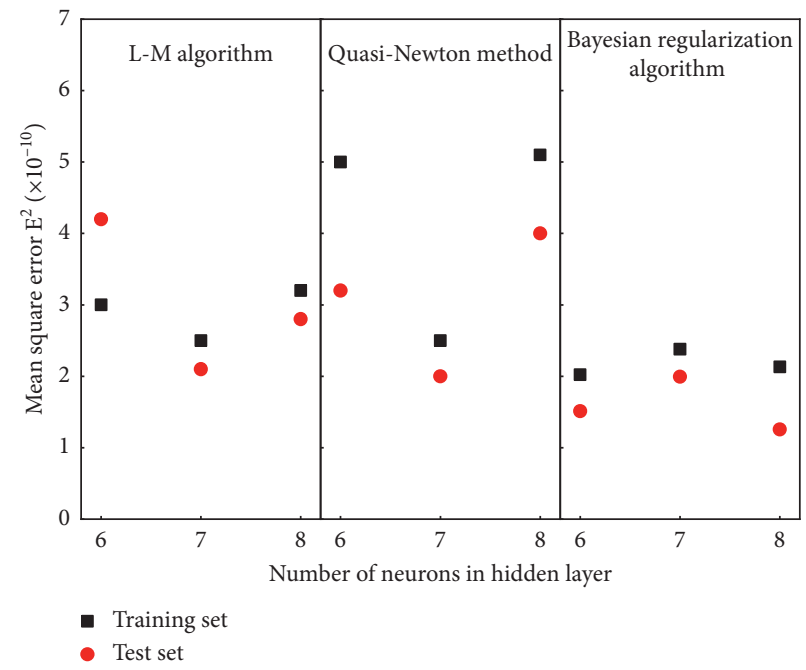

FIGURE 13: Comparison of E2 among different BP neural network models (E2 is the average of 10 prediction results).

making the trained BP neural network dynamically optimal so as to enable them to have both mechanical calculation reliability and practicability of experience judgment.

The training of the BP neural network requires professionals to program, adjust, and update, and the demand for professional knowledge is high, so there is difficulty in wide application and popularization. In order to reduce the learning difficulty and ensure the universality and feasibility, a software for classifying cracked lining structure is developed on the basis of numerical calculation and BP neural network training, as well as the features (such as light weight, 
TABLE 5: Average value of R2 predicted by different training data functions for 10 times.

\begin{tabular}{lccc}
\hline Training algorithm & Number of neurons in hidden layer & Training set $R^{2}$ & Test set $R^{2}$ \\
\hline L-M algorithm & 7 & 0.958 & 0.982 \\
Quasi-Newton method & 7 & 0.947 & 0.953 \\
Bayesian regularization algorithm & 7 & 0.976 & 0.989 \\
\hline
\end{tabular}

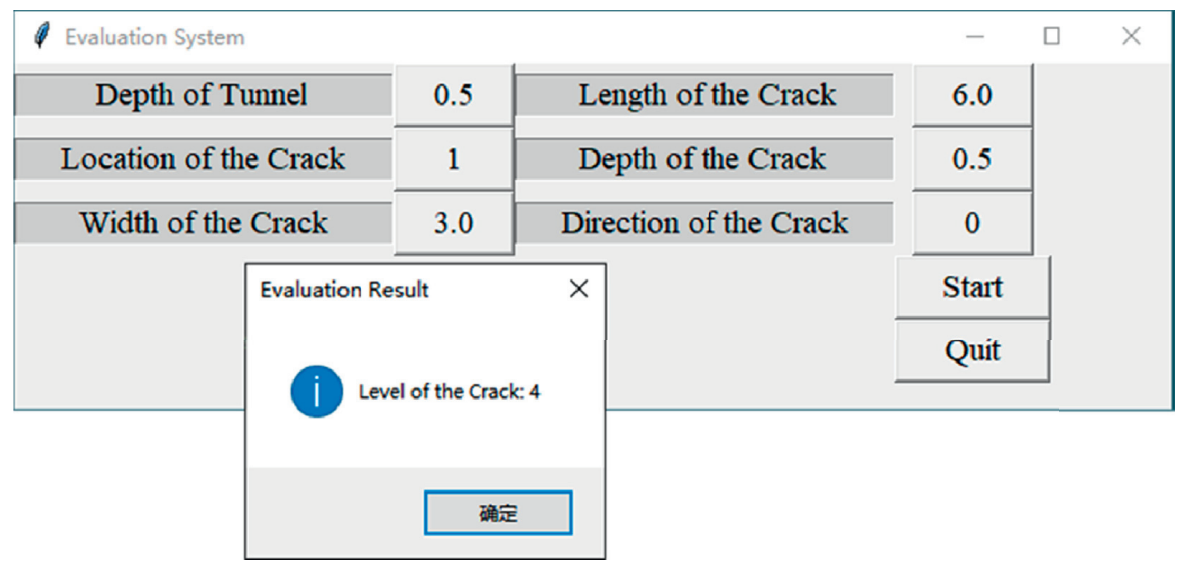

FIGURE 14: Crack classification system based on Python programming and neural network kernel.

high efficiency, and safety) of Python language. Users only need to input crack detection data, and the classification results can be output automatically. The system interface is shown in Figure 14. Through continuous application, update, and optimization, the classification software has already demonstrates good accuracy.

\section{Discussion}

This study aims at crack classification and achieves the accuracy and efficiency of lining crack classification by taking PFC particle flow mechanics calculation as the basis and making use of the BP neural network for training and prediction, supplemented by the expert system to dynamically optimize and adjust the high deviation results. However, due to the following factors, which include the reliability guarantee of using PFC particle flow to calculate and analyze the cracked lining structure, the cause index when calculating the cracked lining and the mechanical characteristics of cracks in the reinforced concrete lining structure is complicated, and further discussion and explanation are elaborated separately as follows:

(1) Reliability guarantee of PFC calculation: compared with traditional finite element analysis or extended finite difference analysis, the selection of PFC calculation parameters involves complex dynamic calibration and optimization, which requires a long adjustment process and a high use cost, so it is relatively less applied. However, as a major challenge affecting engineering safety, it is urgent to seek new analysis ideas and obtain intuitive calculation results to guide engineering disease prevention and control. In addition, the significance far exceeds the cost itself. Based on the existing mechanical test of concrete specimen and the model experiment of bearing characteristics of cracked lining, this project conducts macrosimulation effect controls from both aspects of material characteristics and structural characteristics, thus ensuring the validity and reliability of calculation parameters and calculation results.

(2) Consideration of lining crack causes: the causes of cracks in lining structure are the key external causes that affect the crack state. However, due to the characteristics of tunnel structure, most of the genetic parameters, such as structural bias, stratum dislocation, and hydrostatic pressure, cannot be directly obtained by testing, which need to be refined and judged by combining expert analysis. Too many parameters may lead to extremely complex calculation and make it difficult to realize and control. In this paper, taking the tunnel buried depth, surrounding rock grade, and lining thickness as representatives of external action and structural strength, the crack length, width, depth, and direction as phenomenon indexes, the corresponding relationship between the external action and the internal response is established on mechanics. Due to the strong correlation between the causes and phenomena of diseases, the crack phenomena are very different under different causes of diseases. If the input parameters are contrary to the model assumptions, the fitting results of the BP neural system will deviate greatly from the training data, so expert system intervention is adopted for constantly improving and optimizing the neural network 
parameters, so as to dynamically consider the complex conditions eventually.

(3) Consideration of cracks in reinforced concrete lining: tunnel lining structure is mainly divided into plain concrete and reinforced concrete. In early tunnel construction, due to the inadequate economic development, plain concrete is widely used. However, due to the weak tensile capacity of concrete materials, when the surrounding rock condition is poor, the tunnel lining is prone to bending failure and shear failure under the action of load, thus leads to a lot of cracks in the early plain concrete structure. That is the reason why this study mainly focuses on plain concrete structure. For reinforced concrete structure, the PFC particle flow method in the study of lining cracks is still not mature, the numerical modeling and reduction of the cooperative bearing capacity with different materials of steel and concrete are relatively complex and involve more indexes and parameters. Therefore, the research on the mechanical characteristics and classification of cracks in reinforced concrete structure is included in the next stage of work, and the follow-up research will be carried out continually upon the maturity of conditions.

\section{Conclusion}

This study focuses on the difficult problem of tunnel crack classification, taking plain concrete structure as the object, using the PFC particle flow calculation method to calculate the bearing capacity and crack propagation characteristics of cracked structure under different load conditions, structural conditions, and crack phenomena. The BP neural network system based on mechanical calculation results has been obtained through training. In this study, expert dynamic errata mechanism is introduced and the software is programmed to achieve accurate and efficient fracture classification.

The specific research achievements are as follows:

(1) In this study, the axial compression test of C30 plain concrete structure and the bearing capacity test of cracked tunnel have been carried out. Through the analysis and comparison of bearing capacity and deformation characteristics, the calculation parameters of PFC are dynamically investigated. The errors between the final simulation results of structural displacement, crack depth, and the test results are less than $10 \%$ and $18 \%$, respectively, which proves the feasibility of PFC in tunnel crack calculation and the accuracy of parameter values taken.

(2) Based on the statistical analysis of diseases, practical operation feasibility, and numerical simulation analysis, a classification index system is proposed, which takes the tunnel buried depth $H$ as the load characterization quantity, and the crack position $P$, crack length $L$, crack width $W$, crack depth $D$, and crack direction $A$ as the disease characterization quantities. Combined with the state of load-bearing safety, crack stability, and expert system, the basic data of lining crack classification based on mechanical characteristics are obtained.

(3) A neural network system using seven hidden layer neurons and the Bayesian regularization algorithm is obtained through the BP neural network artificial intelligence prediction tool as well as the training and verification of the basic data of mechanical calculation results. An expert system errata mechanism is introduced to target the high deviation prediction results, which realizes the dynamic optimization, stability, and reliability of complex disease evaluation.

\section{Data Availability}

The (BP neural network training sample) data used to support the findings of this study are currently under embargo while the research findings are commercialized. Requests for data, (6/12 months) after publication of this article, will be considered by the corresponding author.

\section{Conflicts of Interest}

The authors declare that they have no conflicts of interest.

\section{Authors' Contributions}

Hao Ding and Xinghong Jiang contributed equally.

\section{Acknowledgments}

This work was financially supported by the National Key Research and Development Program of China (2017YFC08060010), the Science and Technology Project of Department of Transportation of Yunnan Province (grant no. YJKJ (2018) 22), and The Key Technology Innovation Special of Key Industries of the Chongqing Science and Technology Bureau (grant nos. cstc2019jscx-fxydX0017, cstc2019jscX-gksbX0071, and cstc2019jscx-gksbX0008).

\section{References}

[1] K. Yau, C. Paraskevopoulou, and S. Konstantis, "Spatial variability of karst and effect on tunnel lining and water inflow. A probabilistic approach," Tunnelling and Underground Space Technology, vol. 97, Article ID 103248, 2020.

[2] "Structural health monitoring of a road tunnel intersecting a large and active landslide," Applied Sciences, vol. 7, no. 12, p. 1271, 2017.

[3] D. L. N. d. F. Amorim, S. P. B. Proença, and J. Flórez-López, "Simplified modeling of cracking in concrete: application in tunnel linings," Engineering Structures, vol. 70, pp. 23-35, 2014.

[4] G. Xu, C. He, Z. Chen, C. Liu, B. Wang, and Y. Zou, "Mechanical behavior of secondary tunnel lining with longitudinal crack," Engineering Failure Analysis, vol. 113, Article ID 104543, 2020.

[5] Y.-C. Chiu, C.-H. Lee, and T.-T. Wang, "Lining crack evolution of an operational tunnel influenced by slope instability," Tunnelling and Underground Space Technology, vol. 65, pp. 167-178, 2017. 
[6] J. Z. Xiao, F. C. Dai, Y. Q. Wei et al., "Cracking mechanism of secondary lining for a shallow and asymmetrically-loaded tunnel in loose deposits," Tunnelling and Underground Space Technology, vol. 43, pp. 232-240, 2014.

[7] Y. Kim and A. Bruland, "Analysis and evaluation of tunnel contour quality index," Automation in Construction, vol. 99, pp. 223-237, 2019.

[8] H. Ding, K. Li, Y. Xiayang et al., "Study on the grade evaluation of highway tunnel cracks based on PFC simulation and BP neural network," in Tunnelling and Underground Construction. GSIC 2018, D. Zhang and X. Huang, Eds., Springer, Singapore, pp. 605-611, 2018.

[9] Y. Zhang, Z. Shao, W. Wei, and R. Qiao, "PFC simulation of crack evolution and energy conversion during basalt failure process," Journal of Geophysics and Engineering, vol. 16, no. 3, pp. 639-651, 2019.

[10] H. Haeri, V. Sarfarazi, and Z. Zhu, "Effect of normal load on the crack propagation from pre-existing joints using Particle Flow Code (PFC)," Computers and Concrete, vol. 19, no. 1, pp. 99-110, 2017.

[11] C. Xu, G. Wang, and Q. Zhang, "A new multi-step backward cloud transformation algorithm based on normal cloud model," Fundamenta Informaticae, vol. 133, no. 1, pp. 55-85, 2014.

[12] R. Jiang, F. Dai, Y. Liu, and M. Wei, "An automatic classification method for microseismic events and blasts during rock excavation of underground caverns," Tunnelling and Underground Space Technology, vol. 101, p. 103425, 2020.

[13] S.-C. Lau, M. Lu, and S. T. Ariaratnam, "Applying radial basis function neural networks to estimate next-cycle production rates in tunnelling construction," Tunnelling and Underground Space Technology, vol. 25, no. 4, pp. 357-365, 2010.

[14] B. Liu, R. Wang, G. Zhao et al., "Prediction of rock mass parameters in the TBM tunnel based on BP neural network integrated simulated annealing algorithm," Tunnelling and Underground Space Technology, vol. 95, Article ID 103103, 2020.

[15] J. Ninić and G. Meschke, "Model update and real-time steering of tunnel boring machines using simulation-based meta models," Tunnelling and Underground Space Technology, vol. 45, pp. 138-152, 2015.

[16] X. Zhang, H. Nguyen, X.-N. Bui et al., "Evaluating and predicting the stability of roadways in tunnelling and underground space using artificial neural network-based particle swarm optimization," Tunnelling and Underground Space Technology, vol. 103, Article ID 103517, 2020.

[17] P. A. Cundall and O. D. L. Strack, "Discussion: a discrete numerical model for granular assemblies," Géotechnique, vol. 30, no. 3, pp. 331-336, 2008.

[18] D. E. Rumelhart, G. E. Hinton, and R. J. Williams, "Learning representations by back-propagating errors," Nature, vol. 323, no. 6088, pp. 533-536, 1986.

[19] J. L. Ticknor, H. Hsu-Kim, and M. A. Deshusses, "A robust framework to predict mercury speciation in combustion flue gases," Journal of Hazardous Materials, vol. 264, pp. 380-385, 2014.

[20] A. Antoniou and W. S. Lu, Practical Optimization: Algorithms and Engineering Applications, Springer Publishing Company, Incorporated, New York, NY, USA, 2010.

[21] M. T. Hagan and M. B. Menhaj, "Training feedforward networks with the Marquardt algorithm," IEEE Transactions on Neural Networks, vol. 5, no. 6, pp. 989-993, 2002.

[22] F. D. Foresee and M T. Hagan, "Gauss-Newton approximation to Bayesian learning," in Proceedings of International Conference on Neural Networks (ICNN'97), Houston, TX, USA, June 1997. 